\title{
Spectrums of Opportunistic Infections and Malignancies in HIV-Infected Patients in Tertiary Care Hospital, China
}

\author{
Jiang Xiao`, Guiju Gao`, Yanmei Li, Wen Zhang, Yunfei Tian, Yinxiu Huang, Wenjing Su, Ning Han, Di \\ Yang, Hongxin Zhao*
}

Center for Infectious Diseases, Ditan Hospital, Capital Medical University, Beijing, the People's Republic of China

\begin{abstract}
Background: HIV-related opportunistic infections (Ols) and malignancies continued to cause morbidity and mortality in Chinese HIV-infected individuals. The objective for this study is to elucidate the prevalence and spectrums of Ols and malignancies in HIV-infected patients in the Beijing Ditan Hospital.

Methods: The evaluation of the prevalence and spectrums of Ols and malignancies was conducted by using the clinical data of 834 HIV-infected patients admitted in the Beijing Ditan hospital from January 1, 2009, to November 30, 2012.

Results: The prevalence and spectrums of Ols and malignancies varied contingent on geographic region, transmission routes, and CD4 levels. We found that tuberculosis was most common Ol and prevalence was $32.5 \%$ followed by candidiasis(29.3\%), Pneumocystis pneumonia(PCP)(22.4\%), cytomegalovirus(CMV) infection(21.7\%), other fungal infections(16.2\%), mycobacterium avium complex(MAC)(11.3\%), cryptococcosis(8.0\%), progressive multifocal leukoencephalopathy(PML)(4.4\%), Cerebral Toxoplasmosis(3.5\%) and Penicillium marneffei infection(1.4\%); while Lymphoma(2.9\%), Kaposi's sarcoma(0.8\%) and cervix carcinoma(0.3\%) were emerged as common AIDS-defining malignancies. Pulmonary OI infections were the most prevalent morbidity and mortality in patients in the AIDS stage including pulmonary tuberculosis $(26.6 \%)$ and PCP $(22.4 \%)$. CMV infection(21.7\%) was most common viral infection; Fungal Ols were one of most prevalent morbidity in patients in the AIDS stage, including oral candidiasis $(29.3 \%)$, other fungal infection $(16.2 \%)$, Cryptococcosis $(8.0 \%)$ and Penicillium marneffei infection (1.4\%). We found the low prevalence of AIDS-defining illnesses in central neural system in this study, including progressive multifocal leukoencephalopathy $(4.4 \%)$, cerebral toxoplasmosis $(3.5 \%)$, tuberculosis meningitis $(3.2 \%)$, cryptococcal meningitis $(2.4 \%)$ and CMV encephalitis (1.1\%). In-hospital mortality rate was 4.3 per 100 person-years due to severe Ols, malignancies, and medical cost constraints.

Conclusions: The prevalence and spectrums of Ols, malignancies and co-infections were discussed in this study. It would help increase the awareness for physicians to make a diagnosis and empirical treatment sooner and plan good management strategies, especially in resource limited regions.
\end{abstract}

Citation: Xiao J, Gao G, Li Y, Zhang W, Tian Y, et al. (2013) Spectrums of Opportunistic Infections and Malignancies in HIV-Infected Patients in Tertiary Care Hospital, China. PLoS ONE 8(10): e75915. doi:10.1371/journal.pone.0075915

Editor: Omar Sued, Fundacion Huesped, Argentina

Received May 9, 2013; Accepted August 19, 2013; Published October 25, 2013

Copyright: (c) 2013 Xiao et al. This is an open-access article distributed under the terms of the Creative Commons Attribution License, which permits unrestricted use, distribution, and reproduction in any medium, provided the original author and source are credited.

Funding: Support for this work was provided by: (1) The Twelve-fifth Key Project The Study of Construction of Representative areas for Prevention and Therapy of Fatal Infectious Diseases such AIDS \& Viral Hepatitis in Chaoyang District, Beijing (2012ZX10004-904); (2)Beijing Natural Science Fund The evaluation of association between polymorphism of leptin or adiponectin and NAFLD complicated with AIDS(7112065). The funders had no role in study design, data collection and analysis, decision to publish, or preparation of the manuscript.

Competing interests: The authors have declared that no competing interests exist.

*E-mail:13911022130@163.com

(. These authors contributed equally to this work.

\section{Introduction}

By the end of $2011,740,000$ people were estimated to be living with HIVIAIDS in China[1], HIV led to immunosuppression that allowed Ols and malignancies to cause diseases in HIV-infected patients. Ols led to frequent morbidity and mortality among HIV-infected individuals, and it mostly depended on CD4 levels, types of transmission route and geographic regions.

Despite the availability of National Antiretroviral Treatment Programs (NFATP) in China[2,3], HIV-related clinical diseases (including Ols and malignancies) continued to cause morbidity and mortality in Chinese HIV-infected individuals. Some HIVinfected patients were not aware of HIV infection until Ols became the first indicator of their disease; some patients were 


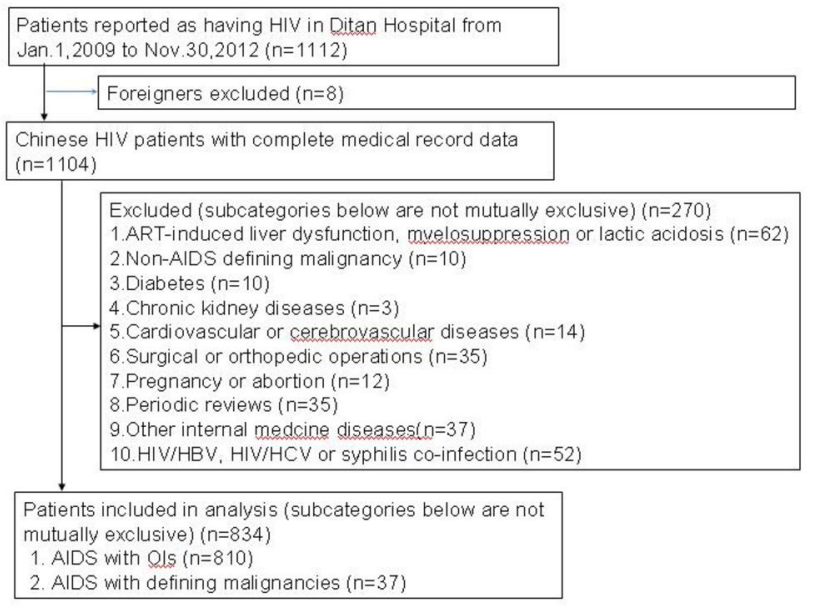

Figure 1. Study flow diagram.

doi: 10.1371/journal.pone.0075915.g001 aware of HIV infection but did not administer antiretroviral(ART) regimens due to skepticism[4] or some social factors; and some HIV-infected patients were taking ART medications but experiencing virological and immunological failure due to poor adherence. HIV-related Ols and malignancies increased morbidity and mortality, which shortened the lifespan of HIVinfected population and increased social-economic burdens on China.

The clinico-epidemiological spectrums of Ols and malignancies in HIV-infected population in China were rarely reported. Therefore it was important to promote awareness of physicians to make a right diagnosis and empirical treatment sooner, in particular, in resource limiting regions. The Beijing Ditan Hospital is a tertiary care hospital in China, which provide high-quality care and treatment to HIVIAIDS patients. The objective for this study is to elucidate prevalence and spectrums of Ols and malignancies in 834 HIV-infected patients in Ditan Hospital.

\section{Methods}

This analysis was approved by the institutional review board (IRB) of Ditan Hospital, the Capital Medical University, Beijing. Per the IRB review, individual informed consent was waived because this analysis used the currently existing data collected during the course of routine treatment and care. The data were reported in aggregate.

\section{Patients selection}

This retrospective observational study was carried out at the Center for Infectious Diseases, Beijing Ditan Hospital, the largest tertiary care hospital for HIVIAIDS patients in North China. The covering area of hospital included most provinces in North China, Northeastern and Northwestern china. The hospital also catered to some patients from provinces in South and Southwestern China.

We reviewed a series of 1112 HIV-infected patients who were admitted to Beijing Ditan Hospital between January1,
2009 and November30, 2012. We excluded some overseas' patients, those diagnosed with non-AIDS-defining illnesses and malignancies, those who co-infected HBV, HCV or syphilis without other Ols or malignancies, the ones who had abortions, pregnant patients, and patients with some internal diseases or who had surgeries(Figure 1). The admitted patients presented some symptoms presumed to be due to Ols and malignancies, who needed evaluation and treatment of HIV-related diseases. Patients completed a face-to-face, paper-and-pencil questionnaire eliciting data on age, gender, ethnicity, marital status, transmission routes and address.

\section{Diagnosis of Ols, malignancies or co-infections}

The diagnosis of Ols and malignancies was taken in accordance with the guideline recommended by the United States Center for Disease Control and Prevention(CDC)[5]. Laboratory tests were performed for blood routine test, liver and kidney functions, serum lipid panels and fasting plasma glucose. The CD4 cell count and percentage were evaluated using FACS calibur flow cytometer. Blood, urine, stool, sputum, bronochoalveolar lavage fluid(BALF) and even cerebrospinal fluid(CSF) were collected and used for culture and identification of species of pathogen depending on patients' symptoms and signs. Fine needle aspiration(FNA) for pathological demonstration was performed in lymphadenopathy and mucocutaneous mass for evaluating aetiology.

Pulmonary tuberculosis was confirmed by the isolation, culture and identification of mycobacterium tuberculosis, or positive acid-fast bacilli(AFB) in sputum or BALF samples. Nucleic acid amplification test was applied to blood or sputum samples, and $\mathrm{y}$-IFN release assay(IGRA) was applied to blood samples to evaluate the patients with clinical presentations who were suspected HIV-related tuberculosis. For patients with symptoms and signs of extrapulmonary tuberculosis, pathological demonstration and AFB in samples of fine needle aspiration of lymph nodes and pleural fluid were required to be performed. The tuberculosis meningitis was diagnosed based on compatible systemic symptoms and signs, and 
cerebrospinal fluid(CSF) analysis indicated pleocytosis with mononuclear predominance, elevated protein and low glucose, and exclusion of cryptococcal meningitis.

The etiologic diagnosis of tuberculosis in HIV-infected patients was difficult, and some patients with negative samples underwent antibiotic treatment for 2 weeks so that bacterial infection could be cured, patients still having fever, cough and night sweat would underwent anti-tuberculosis treatment, tuberculosis was empirically diagnosed due to clinical improvement after empirical anti-tuberculosis treatment.

Oropharyngeal candidiasis were diagnosed based on its features with painless, creamy white, plaque-like lesions in tongue surface or oropharyngeal mucosa which can be scraped off with tongue depressor.

Pneumocystis pneumonia(PCP) was confirmed by compatible clinical symptoms such as subacute onset of progressive dyspnea, fever, nonproductive cough and hypoxemia. Computerized tomography (CT) scan demonstrated patchy ground-glass attenuation. Induced sputum and BALF samples were collected to stain the cyst wall with Gomori methenamine silver. The etiologic diagnosis of PCP in HIV-infected patients sometimes was difficult, and some patients with compatible clinical symptoms and CT results but without positive BALF samples would underwent anti-PCP treatment, PCP was empirically diagnosed due to clinical improvement after empirical anti-PCP treatment.

Cytomegalovirus(CMV) viremia was detected based on CMV PCR and pp65 antigen assay. CMV retinitis was diagnosed according to recognition of characteristic retinal changes during an ophthalmoscopic examination. Endoscopic examination combined with histopathologic identification of intranuclear or intracytoplasmic inclusions was useful tool for diagnosis of CMV colitis. CMV neurologic disease was diagnosed based on compatible neural symtoms and positive CMV-DNA or pp65 antigen assay in CSF.

Detection of serum cryptococcal antigen was used to initially screen cryptococcosis, and patients with positive serum cryptococcal antigen, and with symptoms and signs suggestive of cryptococcal meningitis, should have an lumbar puncture with CSF examination, india ink or CSF cryptococcal antigen assay.

Penicilliosis was confirmed based on isolation and identification of penicilliosis marneffei from blood culture or by histopathologic demonstration of organisms in FNA samples.

The diagnosis of other fungal infection was based on compatible clinical symptoms and measurement of serum $(1,3)-ß-D-g l u c a n[6]$, a cell wall component of most pathogenic fungi, and exclusion of PCP[6]. The culture, isolation and identification of species of fungi in HIV-infected patients were difficult, and some patients with compatible clinical symptoms, positive results of serum (1,3)-ß-D-glucan and exclusion of PCP would underwent anti-fungal treatment, and other fungal infection was empirically diagnosed due to clinical improvement.

Mycobacterium avium complex(MAC) disease was diagnosed based on isolation, culture and identification of MAC from culture of blood, BALF, focal infection sites and bone marrow. MAC infection should be suspected in patients with positive AFB but negative IGRA results who had poor antituberculosis treatment.

Due to lack of brain biopsy, Toxoplasmic encephalitis was empirically diagnosed by clinical symptoms and focal neurological abnormalities, identification of one or more mass lesions by CT scan or magnetic resonance imaging (MRI) of the brain, and seropositive anti-toxoplasma lgG.

AIDS-defining malignancies, including Kaposi's sarcoma, lymphoma and cervix carcinoma were diagnosed based on their characteristic pathological demonstration.

\section{Statistical analysis}

The data were analyzed using SPSS 16.0(SPSS Institute, Chicago IL, USA). The demographic information included age, gender, ethnicity, marital status, transmission routes and address. Descriptive analysis was carried out to evaluate the percentage, mean and standard deviation(SD). The prevalence of Ols and malignancies based on geographic distribution, transmission routes, different CD4 levels and abnormalities in laboratory finding were expressed in percentages. The data were presented as median with interquartile range(IQR) when statistical distribution was skewed. $X^{2}$ test was applied in the prevalence of different Ols between the above and below 200 cells/ul group and $p$ value $<0.05$ was considered statistically significant.

\section{Results}

\section{Demographic profile}

278 patients were excluded from the sample of 1112 patients admitted in Beijing Ditan Hospital from January1, 2009 to November30, 2012. In total, 810 had Ols(72.8\%), and 37 had AIDS-defining malignancies(3.3\%) (See Figure 1).

Of 834 clinical records analyzed (Table 1), 695(82.8\%) were male and $139(17.2 \%)$ were female patients, and the mean age of the study population was $39.2 \pm 11.4$ years. The patients came from different provinces in China, $46.6 \%$ of patients were from North China, followed by $21.5 \%, 17.4 \%, 10.3 \%$ and $4.2 \%$ of the patients from Southern, Northeastern, Southwestern and Northwestern China. The most common transmission route was homosexual contact $(48.7 \%)$, followed by extra-marital heterosexual contact (24.6\%), transfusion(21.3\%), drug addiction(4.1\%) and vertical transmission(1.3\%). CD4 cell counts were calculated in all study population, 494 patients (59\%) had CD4 counts less than 50 cells/ul, followed by $18.0 \%$, $14.4 \%$ and $8.6 \%$ of the patients with $50-100,100-200$ and more than 200 cells/ul, respectively.

Routine blood test results indicated that anaemia was found in $66.9 \%$ of the study population, and neutropenia was present in $39.2 \%$ of these patients. Liver dysfunction was found in $30.4 \%$ of the patients. Serum lipid profile indicated that a number of patients had hyperlipidemia and, $90.3 \%$ of which were with presented with lowered HDL, followed by $35.6 \%$ with increased TG level (Table 2). 
Table 1. Demographic Characteristics and Prevalence of Ols and Malignancies.

\begin{tabular}{|c|c|c|c|}
\hline Characteristics & Total (\%) & Male (\%) & Female (\%) \\
\hline Total patients & $834(100)$ & $695(82.8)$ & 139(17.2) \\
\hline Age(yrs) & $39.2 \pm 11.4$ & $39.1 \pm 11.4$ & $39.4 \pm 11.6$ \\
\hline \multicolumn{4}{|c|}{ Geographic distribution (\%) } \\
\hline Northeast China & $145(17.4)$ & 134(16.1) & $11(1.3)$ \\
\hline Northwest China & $35(4.2)$ & $32(3.9)$ & $3(0.3)$ \\
\hline North China & $389(46.6)$ & $329(39.4)$ & $60(7.2)$ \\
\hline South China & 179(21.5) & 139(16.7) & $40(4.8)$ \\
\hline Southwest China & $86(10.3)$ & $61(7.2)$ & $25(3.1)$ \\
\hline \multicolumn{4}{|c|}{ Transmission-risk categories (\%) } \\
\hline Homosexuality & $406(48.7)$ & $375(45.0)$ & $31(3.7)$ \\
\hline Heterosexuality & $205(24.6)$ & $170(20.4)$ & $35(4.2)$ \\
\hline Drug addiction & $34(4.1)$ & $29(3.9)$ & $5(0.2)$ \\
\hline Transfusion & $178(21.3)$ & $113(13.9)$ & $65(7.4)$ \\
\hline Vertical transmission & $11(1.3)$ & $8(1.0)$ & $3(0.3)$ \\
\hline \multicolumn{4}{|l|}{ CD4 distribution (\%) } \\
\hline CD4 $\leq 50$ cells/ul & $492(59.0)$ & $403(48.3)$ & $89(11.7)$ \\
\hline $50<\mathrm{CD} 4 \leq 100$ cells/ul & $150(18.0)$ & 131(15.7) & $19(2.3)$ \\
\hline $100<\mathrm{CD} 4 \leq 200 \mathrm{cells} / \mathrm{ul}$ & $120(14.4)$ & $100(12.0)$ & $20(2.2)$ \\
\hline CD4>200 cells/ul & $72(8.6)$ & $61(7.3)$ & $11(1.3)$ \\
\hline \multicolumn{4}{|c|}{ AIDS-associated clinical diseases (\%) } \\
\hline Tuberculosis & $271(32.5)$ & $227(27.2)$ & $44(5.3)$ \\
\hline Candidiasis & $245(29.3)$ & $202(24.2)$ & $43(5.1)$ \\
\hline PCP & $187(22.4)$ & $163(19.5)$ & $24(2.9)$ \\
\hline CMV infection & $181(21.7)$ & $148(17.7)$ & $33(4.0)$ \\
\hline Other fungal infection & $135(16.2)$ & $112(13.4)$ & $23(2.8)$ \\
\hline MAC & $94(11.3)$ & $85(10.3)$ & $9(1.0)$ \\
\hline Cryptococcosis & $67(8.0)$ & $53(6.3)$ & $14(1.7)$ \\
\hline PML & $37(4.4)$ & $30(3.6)$ & $7(0.8)$ \\
\hline Cerebral Toxoplasmosis & $29(3.5)$ & $23(2.7)$ & $6(0.8)$ \\
\hline Lymphoma & $27(2.9)$ & $21(2.2)$ & $6(0.6)$ \\
\hline Penicillium Marneffei & $12(1.4)$ & $12(1.4)$ & $0(0.0)$ \\
\hline Kaposi's Sarcoma & $7(0.8)$ & $6(0.6)$ & $1(0.1)$ \\
\hline Cervix carcinoma & $3(0.3)$ & -- & $3(0.3)$ \\
\hline
\end{tabular}

\section{Spectrum of Ols, malignancies and co-infections}

Tuberculosis was most common $\mathrm{Ol}$ among 834 clinical records analyzed(Table 1 ), and the prevalence rate was $32.5 \%$. This was followed by candidiasis( $29.3 \%), \operatorname{PCP}(22.4 \%)$, CMV infection(21.7\%), other fungal infections $(16.2 \%)$, MAC(11.3\%), cryptococcosis(8.0\%), PML(4.4\%), Cerebral Toxoplasmosis(3.5\%) and Penicillium Marneffei infection(1.4\%); Lymphoma(2.9\%), Kaposi's sarcoma(0.8\%) and cervix carcinoma $(0.3 \%)$ were emerged as common AIDSdefining malignancies(See Figure 2).

The percentages of different Ols and malignancies based on etiologic and empirical diagnosis were shown in Figure 2. The distributions of different Ols and malignancies in terms of geographic regions, transmission routes and CD4 levels were shown in Figure 3, 4 and 5. The prevalence and spectrums of Ols varied across different regions in China, fungal infections including penicillium Marneffei infection mainly occurred in the
Southern and Southwestern China; not a single case of penicillium Marneffei infection was found in patients from the Northwestern China, while cerebral toxoplasmosis was more common in Northwestern China than in other part of China. The distribution of different Ols and malignancies also varied regarding different transmission routes. CMV infection, Kaposi's sarcoma, and lymphoma were found mainly in the patients with sexual transmission routes, especially in homosexual contact. Comparatively, various Ols exhibited a higher prevalence in patients with CD4 fewer than 200cells/ul $(p<0.05)$.

Pulmonary Ols were the most prevalent morbidity and mortality cause in patients in the AIDS stage. Among these, tuberculosis(32.5\%) were the most common Ols in respiratory system, in which $8.5 \%$ were etiologically diagnosed based on positive samples, while $24 \%$ were empirically diagnosed based on compatible symptoms and negative samples. PCP $(22.4 \%)$ were the second most common Ols in respiratory system $(9.4 \%$ 
Table 2. Laboratory results in 834 patients with HIVIAIDS.

\begin{tabular}{|c|c|c|c|}
\hline Variable & Range & Mean \pm SD & Abnormality (\%) \\
\hline Neutrophil count $\left(\times 10^{9} / \mathrm{L}\right)$ & $2.00-8.00$ & $4.00 \pm 3.11$ & 39.2 \\
\hline Neutrophil (\%) & $50.00-75.00$ & $68.4 \pm 17.0$ & 54.4 \\
\hline Lymphocyte count $\left(\times 10^{9} / \mathrm{L}\right)$ & $1.00-5.00$ & $0.93 \pm 0.94$ & 66.0 \\
\hline Haemoglobin (g/L) & $120.0-160.0$ & $108.8 \pm 22.9$ & 66.9 \\
\hline PLT count $\left(\times 10^{9} / \mathrm{L}\right)$ & $100.0-300.0$ & $208.8 \pm 101.6$ & 28.9 \\
\hline CD4 cell count (cells/ul) & $706-1125$ & $76.4 \pm 101.1$ & 99.8 \\
\hline $\operatorname{ALT}(\mathrm{U} / \mathrm{L})$ & $0.0-40.0$ & $41.6 \pm 60.3$ & 30.4 \\
\hline AST (U/L) & $0.0-40.0$ & $42.5 \pm 56.5$ & 30.3 \\
\hline T-BIL (umol/L) & $0.0-18.8$ & $10.6 \pm 20.1$ & 6.8 \\
\hline $\operatorname{ALB}(g / L)$ & $35.0-53.0$ & $33.4 \pm 6.9$ & 62.1 \\
\hline BUN (mmol/L) & $1.70-8.30$ & $4.5 \pm 3.2$ & 10.2 \\
\hline $\mathrm{Cr}$ (umol/L) & $59.00-104.00$ & $62.9 \pm 28.6$ & 54.8 \\
\hline TG (mmol/L) & $0.57-1.71$ & $1.6 \pm 1.1$ & 35.6 \\
\hline $\mathrm{TC}(\mathrm{mmol} / \mathrm{L})$ & $2.90-5.68$ & $3.5 \pm 1.1$ & 33.4 \\
\hline $\mathrm{LDL}(\mathrm{mmol} / \mathrm{L})$ & $0.00-3.36$ & $2.1 \pm 0.8$ & 6.8 \\
\hline $\mathrm{HDL}(\mathrm{mmol} / \mathrm{L})$ & $1.09-1.92$ & $0.7 \pm 0.4$ & 90.3 \\
\hline $\mathrm{FPG}(\mathrm{mmol} / \mathrm{L})$ & $4.16-6.44$ & $5.6 \pm 1.9$ & 28.3 \\
\hline
\end{tabular}

PLT: Platelet; ALT: Alanine aminotransferase; AST: Aspartate aminotransferase ; T-BIL: Total Bilirubin; ALB: Albumin; BUN: Blood urea nitrogen; Cr: Serum creatinine; TG: triglyceride ; TC: Total cholesterol; LDL: Low-density lipoprotein; HDL: high-density lipoprotein; FPG: Fasting plasma glucose.

doi: 10.1371/journal.pone.0075915.t002

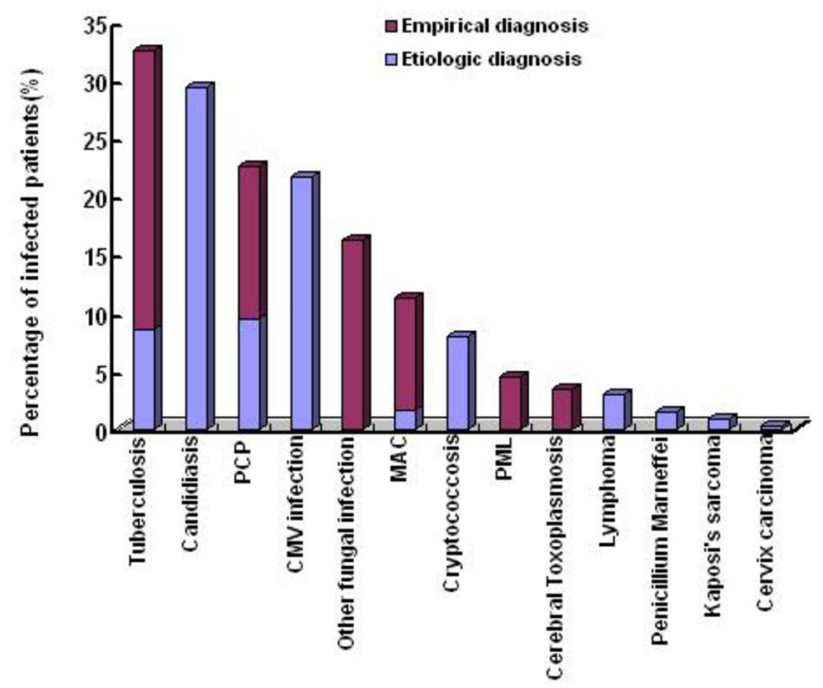

Figure 2. Spectrum of Ols and Malignancies in HIV-infected Patients based on etiologic and empirical diagnosis in the Beijing Ditan Hospital from January 1, 2009 to November 30, 2012. Note: PCP:pneumocystis pneumonia; CMV: cytomegalovirus; MAC: Mycobacterium avium complex; PML: progressive multifocal leukoencephalopathy.

doi: 10.1371/journal.pone.0075915.g002

etiologically diagnosis and $13.0 \%$ empirically diagnosis) (See Figure 2), and PCP was most common cause of death in this study(See Table 3).

Tuberculosis is a systemic disease and it was the most common OI in this study. In total, $26.2 \%$ of these patients were diagnosed with pulmonary tuberculosis while $17.3 \%$ of the patients had extrapulmonary tuberculosis that included lymphoid tuberculosis(8.9\%), pleuritis(6.2\%) and meningitis(3.2\%), in which $11.8 \%$ of patients had pulmonary and extrapulmonary tuberculosis. Pulmonary and meningitis tuberculosis were common causes of death in HIV/tuberculosis co-infection (Table 3).

Viral infection was another prevalent morbidity and mortality cause in patients in the AIDS stage that mainly included CMV infections (Table 3). CMV infection was the most common viral infection and the prevalence rate was $21.7 \%$. $16.4 \%$ of these 


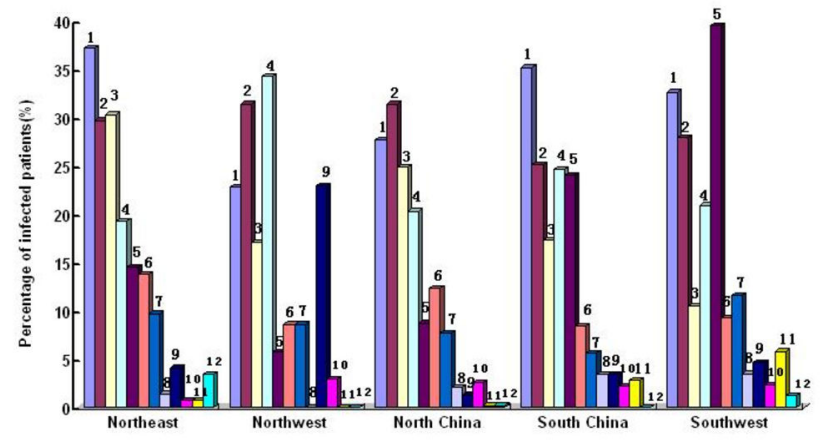

Figure 3. Prevalence of Ols and Malignancies Related to Geographic Regions in the Study Subjects. 1.Tuberculosis; 2.Candidiasis; 3.PCP; 4.CMV infection; 5.Other fungal infection; 6.MAC; 7.Cryptococcosis; 8. PML; 9. Cerebral Toxoplasmosis; 10. Lymphoma; 11.Penicillium Marneffei infection; 12. Kaposi's Sarcoma.

doi: 10.1371/journal.pone.0075915.g003

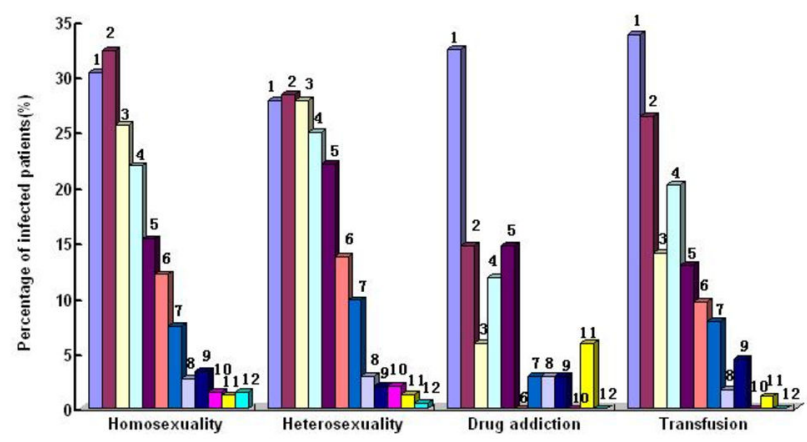

Figure 4. The Prevalence of Ols and Malignancies Related to Transmission Routes in the Study Subjects. 1.Tuberculosis; 2.Candidiasis; 3.PCP; 4.CMV infection; 5.Other fungal infection; 6.MAC; 7.Cryptococcosis; 8. PML; 9. Cerebral Toxoplasmosis; 10. Lymphoma; 11.Penicillium Marneffei infection; 12. Kaposi's Sarcoma.

doi: 10.1371/journal.pone.0075915.g004

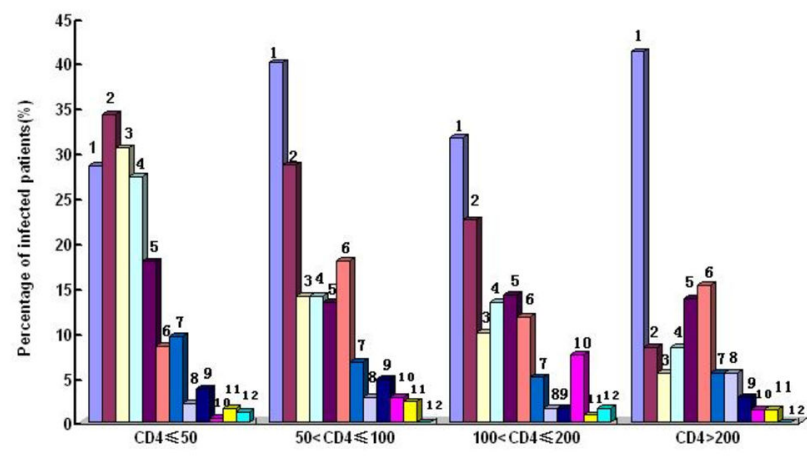

Figure 5. The Prevalence of Ols and Malignancies related to CD4 counts in study subjects. 1.Tuberculosis; 2. Candidiasis; 3.PCP; 4.CMV infection; 5.Other fungal infection; 6.MAC; 7.Cryptococcosis; 8. PML; 9. Cerebral Toxoplasmosis; 10. Lymphoma; 11.Penicillium Marneffei infection; 12. Kaposi's Sarcoma.

doi: 10.1371/journal.pone.0075915.g005

patients with median CD4 counts 43.3 cells/ul presented with CMV viremia without any end-organ diseases, while $4.2 \%$ presented with $\mathrm{CMV}$ retinitis and $1.1 \%$ with encephalitis with median CD4 counts 63.5 and 14.2 cells/ul, respectively. A patient with CD4 count 5 cells/ul was diagnosed with CMV colitis based on endoscopic examination combined with histopathologic demonstration that indicated that CMV-related end-organ diseases often occurred in patients with CD4 counts 
Table 3. AIDS-related Ols and Malignancies and In-hospital Deaths in 834 Patients.

\begin{tabular}{|c|c|c|c|}
\hline Diagnosis & Prevalence (\%) & Median CD4 counts (IQR) & In-hospital deaths $\mathrm{n}(\%)^{1}$ \\
\hline \multicolumn{4}{|l|}{ Tuberculosis } \\
\hline Pulmonary tuberculosis & $222(26.6)$ & $74.5(1-312)$ & $4(2.8)$ \\
\hline Lymphoid tuberculosis & $74(8.9)$ & $67.5(1-276)$ & -- \\
\hline Tuberculous pleuritis & $52(6.2)$ & $89.7(3-351)$ & -- \\
\hline Tuberculous meningitis & $27(3.2)$ & $57.4(3-210)$ & $8(5.6)$ \\
\hline Candidiasis & $245(29.3)$ & $45.3(1-211)$ & -- \\
\hline PCP & $187(22.4)$ & $35.8(1-211)$ & $47(33.1)$ \\
\hline \multicolumn{4}{|l|}{ CMV infection } \\
\hline $\mathrm{CMV}$ retinitis & $34(4.2)$ & $63.5(1-219)$ & -- \\
\hline CMV cephalitis & $9(1.1)$ & $14.2(1-68)$ & $3(2.1)$ \\
\hline CMV colitis & $1(0.1)$ & 5 & $1(0.7)$ \\
\hline CMV viremia & $137(16.4)$ & 43.3(1-230) & -- \\
\hline Other fungal infection & $135(16.2)$ & $57.6(1-278)$ & -- \\
\hline MAC & $94(11.3)$ & $77.3(1-278)$ & -- \\
\hline \multicolumn{4}{|l|}{ Cryptococcosis } \\
\hline Cryptococcal antigenemia & $67(8.0)$ & $50.0(2-289)$ & -- \\
\hline Cryptococcal meningitis & $20(2.4)$ & $49.9(2-218)$ & $9(6.3)$ \\
\hline PML & $37(4.4)$ & 128.6(18-246) & $2(1.4)$ \\
\hline Cerebral Toxoplasmosis & $29(3.5)$ & $55.1(1-210)$ & $3(2.1)$ \\
\hline \multicolumn{4}{|l|}{ Lymphoma } \\
\hline Diffuse large B cell lymphoma & $12(1.4)$ & $105.8(3-208)$ & $3(2.1)$ \\
\hline Bunkitt's lymphoma & $6(0.7)$ & $126.8(12-212)$ & $3(2.1)$ \\
\hline Unclassified lymphoma & $9(1.1)$ & $91.6(5-178)$ & $9(6.3)$ \\
\hline Penicillium Marneffei & $12(1.4)$ & $49.1(4-210)$ & $1(0.7)$ \\
\hline Kaposi's Sarcoma & $7(0.8)$ & $53.6(10-157)$ & -- \\
\hline Cervix carcinoma & $3(0.3)$ & $403(187-611)$ & $1(0.7)$ \\
\hline
\end{tabular}

less than 100cells/ul. This will increase the awareness of physicians to establish diagnosis and perform treatment of CMV-related end-organ diseases.

Fungal Ols were one of most prevalent morbidity in patients in the AIDS stage that included oral candidiasis(29.3\%), other fungal infection(16.2\%), Cryptococcosis(8.0\%) and Penicillium Marneffei infection(1.4\%)(Figure 2 and Table 1).Fungal infection including Penicillium Marneffei infection often emerged in patients living in Southern or southwestern China.

In this study, a few AIDS-defining malignancies were found(Figure 2 and Table 1), viz. diffused large B cell lymphoma(1.4\%), Bukitt's lymphoma(0.7\%), unclassified lymphoma(1.1\%), Kaposi's sarcoma(0.8\%) and cervix carcinoma( $0.3 \%)$, which were diagnosed based on pathological demonstration.

142 patients(4.3 per 100 person-years) died in this study(Figure 6 and Table 3), including 48 in-hospital deaths caused by unexplained infection, malignancies or intracranial space-occupying lesions. In another 94 deaths, most of them were due to PCP (47 patients), central neural system infection (22 patients), AIDS-defining malignancies (16 patients). 117 patients who died in hospital had CD4 counts less than 100 cells/ul.

\section{Discussion}

In China, HIV-infected patients was treated based on NFATP, which was administered through a low-technology, community-based treatment model[3,4], while Ols and malignancies were treated in HIVIAIDS centers of tertiary care hospital due to poor expertise and facilities in community level hospital. The Beijing Ditan Hospital is the largest center for HIVIAIDS diagnosis and treatment and the cover area included all provinces in China. In this retrospective observational study, 834 HIV-infected patients came from different geographic regions in China and hence the spectrum of Ols and malignancies in current study may apply to different regions in China.

An accurate evaluation of the prevalence and spectrum of Ols and malignancies helps to plan good management strategies. It was reported that[7], in a series of 135 hospitalized HIV-infected patients from north India, the five most common AIDS-defining illnesses were tuberculosis(71\%), candidiasis(39.3\%), $\quad \operatorname{PCP}(7.4 \%), \quad$ cryptococcal mentingitis(3.7\%) and toxoplasmosis(3.7\%). Another retrospective study in 89 cases in Lebanon with Ols reported[8] that the most common Ols were cerebral toxoplasmosis(21\%) 


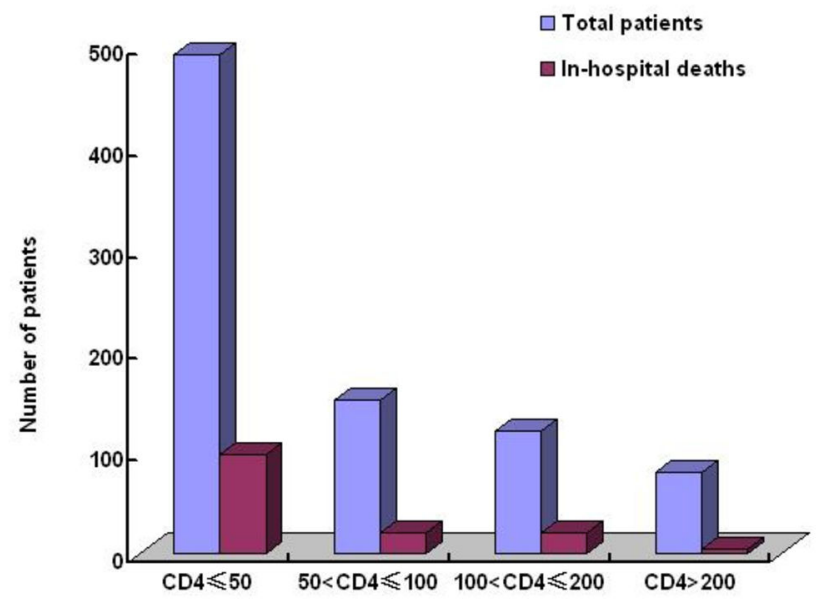

Figure 6. Distribution of CD4+T Counts and In-hospital deaths in the Study Subjects. doi: 10.1371/journal.pone.0075915.g006

and fungal infection(17\%), which indicated that the prevalence and spectrum of Ols in HIV-infected population varied contingent on geographic locations and social-economic conditions. In this study, we found that most common Ols were tuberculosis, candidiasis, PCP, CMV infection, other fungal infection, MAC and Cryptococcosis.

In this study, the majority of the patients $(54.9 \%)$ were in the age group of 20-40 years, and men were predominantly affected, which was consistent with the findings in Indian[9] and Euro-American[10] studies. Thus Ols and malignancies occurred in economically productive years in these HIVinfected individuals that increased social-economic burden in China.

Ols occurred in HIV-infected patients were contingent on geographic locations, and the frequencies and types of Ols varied from region to region due to different climates and social-economic conditions. In this study, HIV-infected patients came from different regions in China, and the findings revealed that other fungal infection[11] and Penicillium Marneffei infection[12] were more common in Southern and Southwestern China, where the ecological conditions and humid climate were similar to that of Southeast Asia. But these fungal infections were rarely found in the northwestern China due to dry climate. We also found that HIV-infected patients in Northwestern China showed higher prevalence of Cerebral Toxoplasmosis than that found in other parts of China. NorthWestern China is live-stock breeding area and therefore, HIVinfected patients were more prone to have Protozoan toxoplasma gondiin infection through contacting with animals.

The most common risk of HIV transmission in this study was homosexual contact, found in $46.9 \%$ of patients, and this was followed by non-marital heterosexual contact (25.4\%), which indicated that sexual contacts had become the most common transmission route of HIV infection in China. The findings by transmission routes revealed that Ols and malignancies such as CMV infection, lymphoma and Kaposi's sarcoma were more common in sexual contacts. It was reported [13] that CMV infection and Kaposi's sarcoma occurred in patients who had contacted with men who have sex with $\operatorname{men}(\mathrm{MSM})$, and intravenous users that was similar to that of the findings in this study.

The findings in this study revealed that prevalence of transfusion transmission was $21.3 \%$. It was reported [14] that former plasma donors across central China sold plasma to unscrupulous collectors in unsanitary conditions during the early-mid-1990s, resulting in untold numbers of HIV infection. Chinese government had banned paid blood donation since 2000 and free antiretroviral therapy in NFATP was provided to former plasma donors to control HIV transmission. Patients who had blood transfusion during the early-mid-1990s were not aware of HIV infection until Ols or malignancies became the first indicator of their disease. In this study, $21.3 \%$ of patients had blood transfusion during the early-mid-1990s, got Ols or malignancies after a decade of latent period.

The culture, isolation and identification of species of pathogens such as mycobacterium tuberculosis, Pneumocyst, some invasive fungi and Mycobacterium avium complex in some HIV-infected patients were difficult, and due to lack of brain biopsy, pathological diagnosis of Toxoplasmic encephalitis and PML was also difficult, empiric diagnosis and treatment was taken in patients with compatible clinical and radiographic results, which helped us minimize the false negative diagnosis.

The important finding in this study revealed that pulmonary Ol infections were the most prevalent morbidity cause in patients in the AIDS stage, and tuberculosis and PCP were most common Ols in respiratory system, which was similar with results in Indian [15]. The process of diagnosis of pulmonary OI infections was well planned, and HIV-infected patients with symptoms in respiratory tract underwent mycobacterium tuberculosis culture in induced sputum and BALF samples, pathological demonstration and $\mathrm{PCR}$ detection for $P C P$ or mycobacterium tuberculosis in BALF samples, IGRA assay[16] for mycobacterium tuberculosis and thin-section computerized tomography scan. Empiric diagnosis and anti-tuberculosis treatment was taken in some patients with negative samples who were suspected of being infected with tuberculosis. 
The prevalence of mycobacterium tuberculosis infection was $32.5 \%$ in this study, which was similar to reports in Indian studies [17]. Tuberculosis infection was a systemic disease, and with increasing degrees of immunodeficiency, extrapulmonary tuberculosis was more common. In this study, we found that pulmonary tuberculosis was the most widespread $(26.6 \%)$ followed by lymphadenitis $(8.9 \%)$, pleuritis $(6.2 \%)$ and meningitis (3.2\%), in which $11.8 \%$ of patients had pulmonary and extrapulmonary tuberculosis. This indicated that that tuberculosis infection was one of most widespread Ols in Chinese HIV-infected patients. We also found that tuberculosis infection was prevalent at any levels of CD4 counts. These findings indicated that appropriate tuberculosis management strategies should be planned and implemented in Chinese HIVinfected population.

PCP was found in $22.4 \%$ of patients in this study, which was diagnosed based on etiology and empirical treatment. Pneumocystis jirovecii cannot be cultivated routinely, cytopathologic demonstration of this organism in BALF was required for etiologic diagnosis. Empirical diagnosis was based on compatible clinical symptoms and $\mathrm{CT}$ results, negative cytopathologic demonstration of organism and clinical improvement after empirical anti-PCP treatment. we found that prevalence of etiologic and empirical diagnosis were $9.4 \%$ and $13.0 \%$, respectively. serum $(1,3)-ß-D$-glucan may be elevated in patients with $\mathrm{PCP}$, but the assay 's sensitivity and specificity for establishing a PCP diagnosis were problematic [18]. In this study, we did not adopt elevated serum $(1,3)-\beta-D-g l u c a n$ as diagnostic standard for PCP.

The findings in this study revealed that CMV infection was the most common viral infection. Most of CMV infection, especially CMV related end-organ diseases, occurred in HIVinfected patients with immune suppression, typically in those with CD4 count less than 50cells/ul [19]. In this study, $21.7 \%$ of the patients were found to have CMV infection, in which $4.2 \%$ and $1.1 \%$ had found retinitis and cephalitis, and the median CD4 counts were 63.5 cells/ul and 14.2 cells/ul, respectively. Endoscopic examination combined with biopsy and histopathologic examination in HIV-infected patients with digest tract symptoms was performed and one case of CMV colitis had been found.

The occurrence of oral candidiasis was recognized as an indicator of immune suppression, and those were often found in HIV-infected patients with CD4 counts fewer than $200 \mathrm{cells} / \mathrm{ul}$. The prevalence of oral candidiasis in this study was 29.3\% with the median CD4 counts 45.3cells/ul, which indicated that the occurrence of oral candidiasis helped physicians in resource constrained regions in China to diagnose the clinical progression of HIV infection.

The findings in this study revealed that prevalence of Penicillium Marneffei infection was $1.4 \%$. Penicilliosis was known to be endemic in Southeast Asia and southern China, and the definitive diagnosis of penicilliosis was based on culture, isolation and identification of species of Penicillium Marneffei from blood samples or other clinical specimens. We found that penicilliosis was mainly diagnosed in patients from southern or southwestern China, and most cases of penicilliosis were observed in patients who had CD4 cell counts less than 100cells/ul(mean CD4 cell counts was 49.1cells/ul), This will increase the awareness of physicians to establish diagnosis and perform treatment of penicilliosis in patients with less than 100cells/ul from southern or southwestern China.

Except Candidiasis, Penicillium Marneffei, Cryptococcosis, The culture, isolation and identification of species of other fungi in HIV-infected patients were difficult, and some patients with compatible clinical symptoms, positive results of serum $(1,3)-ß-$ D-glucan and exclusion of PCP was empirically diagnosed as other fungal infection, and the prevalence was $16.2 \%$ in this study. Other fungal infection was diagnosed with $(1,3)-B-D-$ Glucan assay with standard reference value defining $>100 \mathrm{pg} / \mathrm{ml}$, which should be differentiated with PCP. It was reported [6] that (1,3)-ß-D-Glucan was correlated with HIVrelated $\mathrm{PCP}$. In this study, PCP was diagnosed based on characteristic clinical presentation, thin-section computerized tomography scan and histopathological demonstration pneumocystis jirovecii in BALF, but not on elevated serum $(1,3)-ß-D-g l u c a n$ while other fungal infection was diagnosed based on systemic symptoms and (1,3)-ß-D-Glucan $>100 \mathrm{pg} / \mathrm{ml}$.

We found the low prevalence of AIDS-defining illnesses in central neural system in this study, including PML $(4.4 \%)$, cerebral toxoplasmosis $(3.5 \%)$, tuberculosis meningitis $(3.2 \%)$, cryptococcal meningitis $(2.4 \%)$ and CMV encephalitis (1.1\%), which contributed to increased mortality in HIV-infected patients. Lubar puncture and MRI scan were required in HIVinfected patients with central nervous system (CNS) manifestation and different pathogen detections were performed in cerebrospinal fluid to differentiate various OI infections. We found that etiologic diagnosis of intracranial space-occupying lesions were most difficult in this study. Due to high risk and poor adherence, we hardly took brain biopsy in these patients. space-occupying lesions were empirically diagnosed based on clinical symptoms, focal neurological abnormalities and identification by CT scan or magnetic resonance imaging(MRI) of the brain, which may be the reason for low prevalence of Ols and malignancies in central neural system in this study.

We also found some AIDS-defining malignancies including lymphoma, Kaposi's saroma and cervix carcinoma, which were definitively diagnosed based on pathological demonstration. Fine needle aspiration combined with pathological demonstration was performed in lymphadenopathy and mucocutaneous mass, which helped physicians to differentiate malignancies with $\mathrm{OI}$ infections. We found that incidence ratio of AIDS-related Kaposi's saroma and cervix carcinoma in this study was similar with that reported in Euro-American[20,21], while prevalence of lymphoma was lower than that in EUROSIDA cohort[22], which indicated that pathogenicity of lymphoma should be further understood in HIV-infected patients.

In-hospital mortality in this study was 4.3 per 100 personyears, and 94 of these deaths were due to severe Ols and malignancies while 48 were caused by unexplained infections or malignancies and intracranial space-occupying lesions due to cost constraints and unaffordable further diagnosis and treatment. 
Our study has some additional limitations. First, the study is not a meta-cohort observational analysis in this cohort, with the increase in the number of patients in the observational cohort, the results achieved in this study should remain to be further validated. The second limitation is inherent biases based on observational data (i.e. bias such as referral of complex patients from other centers, or patients dying before arriving the hospital might increase or reduce the frequency of admissions) and without control group for comparison.

In conclusion, the prevalence and spectrums of Ols and malignancies varied contingent on geographic region, transmission route and CD4 level. Tuberculosis, candidiasis, $\mathrm{PCP}, \mathrm{CMV}$ infection, other fungal infection and MAC emerged as the most common Ols. In-hospital mortality rate was considerable due to severe Ols, malignancies and cost constraints. It would help to increase the awareness of physicians to come up with right diagnoses and implement empirical treatment sooner and plan good management strategies, especially in resource constrained regions in China.

\section{References}

1. UNAIDS/WHO Working Group on HIV Global/AIDS and STI Surveillance. (2012) China's epidemic \& response, 2012 update. Available: http://www.unaids.org.cn/en/index/index.asp.

2. Zhang FJ, Pan J, Yu L, Wen Y, Zhao Y (2005) Current progress of China's free ART program. Cell Res 15: 877-882. doi:10.1038/sj.cr. 7290362. PubMed: 16354563.

3. Zhang F, Haberer JE, Wang Y, Zhao Y, Ma Y et al. (2007) The Chinese free antiretroviral treatment program: challenges and responses. AIDS 21 Suppl 8: S143-S148. doi:10.1097/01.aids.0000304710.10036.2b. PubMed: 18172383.

4. Chou IH (2004) China's new AIDS policy faces great wall of skepticism. Nat Med 10: 4. doi:10.1038/nm0104-4a. PubMed: 14702606.

5. Panel on Opportunistic Infections in HIV-Infected Adults and Adolescents (2013) uidelines for the prevention and treatment of opportunistic infections in HIV-infected adults and adolescents: recommendations from the centers for disease control and prevention, the National Institutes of Health, and the HIV Medical Association of the Infectious Diseases Society of America. Available: http:// aidsinfo.nih.gov/contentfiles/lvguidelines/adult oi.pdf. Accessed 2013 May 7.

6. Sax PE, Komarow L, Finkelman MA, Grant PM, Andersen J et al. (2011) Blood1,3)-beta-D-glucan as a diagnostic test for HIV-related Pneumocystis jirovecii pneumonia. Clin Infect Dis 53:197-202.

7. Sharma SK, Kadhiravan T, Banga A, Goyal T, Bhatia I et al. (2004) Spectrum of clinical disease in a series of 135 hospitalised HIV-infected patients from north India. BMC Infect Dis 4: 52 . doi: 10.1186/1471-2334-4-52. PubMed: 15555069.

8. Naba MR, Kanafani ZA, Awar GN, Kanj SS (2010) Profile of opportunistic infections in HIV-infected patients at a tertiary care center in Lebanon. J Infect Public Health 3:130-133.

9. Srirangaraj S, Venkatesha D (2011) Opportunistic infections in relation to antiretroviral status among AIDS patients from south India. Indian. J Med Microbiol 29: 395-400. doi:10.4103/0255-0857.90175. PubMed: 22120801.

10. Severe P, Leger P, Charles M, Noel F, Bonhomme G et al. (2005) Antiretroviral therapy in a thousand patients with AIDS in Haiti. N Engl J Med 353: 2325-2334. doi:10.1056/NEJMoa051908. PubMed: 16319381.

11. Chaiwarith $R$, Fakthongyoo A, Praparattanapan J, Boonmee $D$, Sirisanthana T et al. (2011) Itraconazole vs fluconazole as a primary prophylaxis for fungal infections in HIV-infected patients in Thailand

\section{Acknowledgements}

We acknowledge the work of HIV health care providers for their diagnosis, nursing and treatment of HIVIAIDS patients in Ditan Hospital. We acknowledge the work of social workers and volunteers in Beijing Red Ribbon who provide counseling, adherence interventions and resolving psychosocial issues for HIVIAIDS patients.

\section{Author Contributions}

Conceived and designed the experiments: HZ. Performed the experiments: JX. Analyzed the data: JX GG WS. Contributed reagents/materials/analysis tools: YL WZ YT YH NH DY WS. Wrote the manuscript: JX.
Curr HIV Res 9: 334-338. doi:10.2174/157016211797635991. PubMed: 21916838.

12. Chaiwun $B$, Vanittanakom $N$, Jiviriyawat $Y$, Rojanasthien $S$, Thorner $P$ (2011) Investigation of dogs as a reservoir of Penicillium marneffei in northern Thailand. Int J Infect Dis 15: e236-e239. doi:10.1016/j.ijid. 2010.12.001. PubMed: 21236714.

13. Jones JL, Hanson DL, Dworkin MS, Kaplan JE, Ward JW (1998) Trends in AIDS-related opportunistic infections among men who have sex with men and among injecting drug users, 1991-1996. J Infect Dis 178: 114-120. doi:10.1086/515593. PubMed: 9652430.

14. Dou Z, Chen RY, Wang Z, Ji G, Peng G et al. (2010) HIV-infected former plasma donors in rural Central China: from infection to survival outcomes, 1985-2008. PLOS ONE.5: e13737. doi:10.1371/ journal.pone.0013737. PubMed: 21060835.

15. Chakraborty N, Mukherjee A, Santra S, Sarkar RN, Banerjee D et al. (2008) Current trends of opportunistic infections among HIVseropositive patients from Eastern India. Jpn. J Infect Dis 61: 49-53.

16. Metcalfe JZ, Everett CK, Steingart KR, Cattamanchi $A$, Huang $L$ et al. (2011) Interferon-y release assays for active pulmonary tuberculosis diagnosis in adults in low- and middle-income countries: systematic review and meta-analysis. J Infect Dis 204 Suppl 4:S1120-1129.

17. Saha K, Firdaus R, Santra P, Pal J, Roy A et al. (2011) Recent pattern of Co-infection amongst HIV seropositive individuals in tertiary care hospital, Kolkata. Virol J 8: 116. doi:10.1186/1743-422X-8-116. PubMed: 21396133

18. Pisculli ML, Sax PE (2008) Use of a serum beta-glucan assay for diagnosis of HIV-related Pneumocystis jiroveci pneumonia in patients with negative microscopic examination results. Clin Infect Dis 46: 1928-1930. doi:10.1086/588564. PubMed: 18540807.

19. Jabs DA, Van Natta ML, Kempen JH, Reed Pavan P, Lim Jl et al. (2002) Characteristics of patients with cytomegalovirus retinitis in the era of highly active antiretroviral therapy. Am J Ophthalmol 133: 48-61. doi:10.1016/S0002-9394(01)01322-8. PubMed: 11755839.

20. Armstrong AW, Lam KH, Chase EP (2013) Epidemiology of classic and AIDS-related Kaposi's sarcoma in the USA: incidence, survival, and geographical distribution from 1975 to 2005. Epidemiol Infect 141: 200-206. doi:10.1017/S0950268812000325. PubMed: 22404880.

21. Abraham AG, Strickler HD, Jing Y, Gange SJ, Sterling TR et al. (2012) Invasive cervical cancer risk among HIV-infected women: A North American multi-cohort collaboration prospective study. J Acquir Immune Defic Syndr [Epub ahead of print]. PubMed: 23254153.

22. Mocroft A, Katlama C, Johnson AM, Pradier C, Antunes F et al. (2000) AIDS across Europe, 1994-98: the EuroSIDA study. Lancet 356: 291-296. doi:10.1016/S0140-6736(00)02504-6. PubMed: 11071184. 